\title{
EFFECT OF GRAIN BOUNDARY MELTING ON SLIDING IN BICRYSTALS: A MOLECULAR DYNAMICS STUDY
}

\author{
P. Deymier and G. Kalonji \\ Department of Materials Science and Engineering \\ Massachusetts Institute of Technology \\ Cambridge, Massachusetts 02139 \\ (Received Ju1y 18, 1985) \\ (Revised October 11, 1985)
}

\section{Introduction}

Grain boundaries are known to exert a profound influence on the properties of polycrystalline materials at high temperature. Experimental studies on grain boundary sliding in Al bicrystals (1), or more recently in $\mathrm{Zn}$ bicrystals (2), show a change in the temperature dependence of the sliding rate at temperatures between $70 \%$ to $90 \%$ of the bulk melting point. The critical temperature at which a change in activation energy for sliding occurs depends to a large extent on the grain boundary type. Coincidence grain boundaries exhibit the highest critical temperatures. It has been suggested that the observed change in the temperature dependence of the sliding rate may be caused by a grain boundary phase transition taking place at the critical temperature (2). The exact nature of this grain boundary phase transition is, however, still unresolved. Many difficulties associated with the small size of the grain boundary region make the experimental investigation of grain boundary phase transitions cumbersome. On the other hand, atomistic computer simulation has been proved to be a very handy technique for the correlation of macroscopic properties to structural properties. In this letter we present molecular dynamics results on grain boundary sliding in a three dimensional Lennard-Jones bicrystal.

\section{Molecular dynamics results}

We have used a non-conventional molecular dynamics formulation with periodic border conditions (3) where the borders of the simulated system are dynamical variables and are driven by the imbalance between an externally applied stress and the internal stress tensor. With this method the simulation cell is allowed to change its shape as well as its volume. This method is therefore particularly suited to the simulation of processes at constant stress. We have studied the response, by grain boundary sliding, of a bicrystal under an applied shear stress parallel to the grain boundary plane. The bicrystal is constituted of 648 particles interacting with each other through a Lennard-Jones (12-6) potential:

$$
\Phi(r)=4 \varepsilon\left\{(\sigma / r)^{12}-(\sigma / r)^{6}\right\}
$$

The interatomic potential was truncated between the third and fourth nearest neighbours. The grain boundary is a coincidence $\Sigma=7$ tilt boundary $(\theta=38.21)$ with tilt axis parallel to the $\langle 111\rangle$ direction. The applied stress tensor combines a shear component and an isostatic pressure. The stress tensor in the fixed reference system $(X, Y, Z)$ of Figure 1 is given by:

$$
\begin{array}{rrr}
-0.1 & 0.2 & 0.0 \\
0.2 & -0.1 & 0.0 \\
0.0 & 0.0 & -0.1
\end{array}
$$

where the reduced components of the stress tensor are related to the components of the stress by $\mathrm{S}_{\mathrm{ij}}{ }^{*}=$ $\mathrm{S}_{\mathrm{ij}} \sigma^{3} / 4 k \varepsilon$. The $(\mathrm{X}, \mathrm{Y}, \mathrm{Z})$ coordinate system is related to the crystallographic reference system $\left(\mathrm{X}^{\prime}, \mathrm{Y}^{\prime}, Z^{\prime}\right)$ by :

$$
\begin{aligned}
& X=(1 / \sqrt{14}) X^{\prime}+(2 / \sqrt{ } 14) Y^{\prime}-(3 / \sqrt{ } 14) Z^{\prime} \\
& Y=(5 / \sqrt{42}) X^{\prime}-(4 / \sqrt{ } 42) Y^{\prime}-(1 / \sqrt{ } 2) Z^{\prime} \\
& 13 \\
& 0036-9748 / 86 \$ 3.00+.00 \\
& \text { Copyright } \quad \text { (c) } 1986 \text { Pergamon Press Ltd. }
\end{aligned}
$$




$$
Z=(1 / \sqrt{ } 3) X^{\prime}+(1 / \sqrt{ } 3) Y^{\prime}+(1 / \sqrt{ } 3) Z^{\prime}
$$

The applied shear stress is approximately $3 \%$ of the shear modulus of argon near the bulk melting temperature (4). We have applied this shear stress to a three dimensional perfect crystal containing 108 particles at the temperature $\mathrm{T}^{*}=0.095$ to make sure that the shear stress would not initiate plastic deformation in a simulated perfect crystal. The perfect crystal showed only elastic deformation. The amount of sliding of one crystal with respect to the other one is measured as the relative shift of the centers of gravity of two slabs, each in the bulk region on either side of the grain boundary as shown in Figure 1. To avoid the contribution of uniform shear deformation to the relative shift, the coordinates of the centers of gravity are calculated in the moving reference system of the borders of the simulation cell. To access the sliding velocity, we monitor the time dependence of the amount of sliding. As pointed out by Andersen (5) the relaxation time for recovery from an imbalance between the external stress and the internal stress is not an absolute time but depends on an arbitrary constant $\mathrm{W}$ introduced in the molecular dynamics formulation as the mass of the borders. Nonequilibrium properties will therefore be functions of $\mathrm{W}$ and the magnitude of the sliding rate we measure cannot be compared to any experimental sliding velocity.

We performed a set of simulations at different temperatures from $34 \%$ up to $88 \%$ of the bulk melting point. At approximately $50 \%$ of the bulk melting temperature, the volume and the enthalpy of the bicrystal versus temperature curves show a singularity followed by a marked change in heat capacity. The $\Sigma=7$ grain boundary is known to undergo a grain boundary phase transition at high temperature. We have reported evidence for a first order melting transition of a $\Sigma=7$ grain boundary in a three dimensional Lennard-Jones bicrystal (6). In this transition the crystalline grain boundary is replaced by a highly disordered liquid-like layer. This melted layer is a few atomic distances thick. The grain boundary melting took place, for the $\Sigma=$ 7 boundary, at $76 \%$ of the bulk melting temperature, for the case of a bicrystal under a reduced isostatic pressure $\mathrm{P}^{*}=0.1$. Figure 2 shows the radial distribution functions of the sheared bicrystal below and above the transition temperature. Below the transition temperature, the radial distribution is characteristic of a crystalline structure. Above the transition temperature, however, the asymmetry of the second neighbours peak suggests some local structural change. We have superposed on the high temperature radial distribution of the bicrystal the radial distribution of a perfect liquid at a reduced scale of $1 / 6$, corresponding to the approximate ratio of atoms in the liquid-like layer and atoms in the crystalline regions. The asymmetry suggests a local melting at the grain boundary involving $1 / 6$ of the particles constituting the sheared bicrystal.

We note that the application of a shear stress of high magnitude reduced the grain boundary melting temperature. The sliding that we observed at the grain boundary occurred in the direction perpendicular to the direction of the applied shear stress as well as in the direction of the applied stress. The ratio of the amount of sliding in the $\mathrm{Z}$ direction to the amount of sliding in the $\mathrm{X}$ direction is approximately two to one above the transition temperature. At low temperature there is no measurable sliding in the $\mathrm{X}$ direction. The sliding in this transverse direction provides an easy sliding path compared to the $X$ direction alone as can be seen on Figure 3 . The total amount of sliding of a crystalline bicrystal near the transition temperature stays below $10 \%$ of an atomic spacing. At high temperature, the amount of sliding reaches $80 \%$ of an atomic separation. We have plotted the logarithm of the total sliding velocity versus the inverse of the temperature in Figure 4 . The temperature dependence of the sliding rate does not show a definitive change at the transition temperature which is approximately $50 \%$ of the bulk melting point. The computer simulation results appear, therefore, to be in disagreement with the experimental results on the temperature dependence of the grain boundary sliding velocity. However, one has to be very careful in comparing computer simulations and experiments because of the difference in time scales. Each simulation in this study lasted approximately 40 atomic vibrational periods, which is an extremely small amount of time compared to the times involved in real experiments. The time length of the simulations is limited by the available computational resources. Therefore, at low temperature, the sliding velocity we measure does not correspond to the sliding velocity of steady state sliding but only to a transient velocity and thus cannot be compared to velocities measured over hours. At high temperatures the steady state sliding is established very rapidly over a short period of time. If we consider at low temperature only the two points closer to the transition temperature, which might more accurately reflect the steady state sliding in a crystalline boundary, the temperature dependence of the sliding velocity shows a change in slope at the transition temperature. The question of the accuracy of the measurements of sliding velocity must be addressed. At high temperature the average error on the sliding velocity is approximately $1 \%$. At low temperature we are not able to characterize it but it certainly increases as the temperature decreases.

The characterization of the grain boundary phase transition through its effect on the mechanical properties of a bicrystal under a shear stress remains in an uncertain state. The grain boundary transition was seen through the thermodynamic properties and the structural properties of the bicrystal. We observed that a shear stress applied to a bicrystal reduces the grain boundary melting temperature. Sliding in the sheared bicrystal was shown to occur preferentially at the grain boundaries. However, the sliding velocity measured in the simulation did not exhibit a clear cut change at the transition temperature. We point out that the length of the simulations corresponds to an 
extremely short time compared to the times involved in experimental measurement of bicrystal sliding velocities. At very low temperature, the sliding velocity measured in a simulation does not describe the steady state sliding, but the sliding velocity before the steady state process is established. We also note that the initial values of the sliding velocity are greater than the values measured at steady state. Because at low temperatures the time needed to attain the steady state sliding velocity is greater, the low temperature data should, if longer simulation times were available, tend to lower values, thereby increasing the slope change at the transition temperature. Experimental results on the temperature dependence of the grain boundary sliding velocity might be reproduced more satisfactorily by running longer simulations.

\section{Acknowledgements}

This work was supported by a Presidential Young Investigator Award. The computing was performed at the Max-Planck Institut fur Metallforschung, Institut fur Werkstoffwissenschaften, Stuttgart. We would like to thank Manfred Ruhle, of that institute, for his hospitality and Siegfried Schmauder for computational assistance. We thank Reza Najafabadi and Craig Counterman for helpful comments.

\section{References}

1. M. Biscondi and C. Goux, Mem. Scient. Revue Metall. 65, 167 (1968).

2. T. Watanabe, S. Kimura and S. Karashuma, Phil. Mag. A 49, 845 (1984).

3. M. Parrinello and A. Rahman, J. Appl. Phys. 52, 7182 (1981)

4. G. Simmons and H. Wang, Single Crystal Elastic Constants and Calculated

Aggregate Properties, M.I.T. Press, Cambridge (1971)

5. H.C. Andersen, J. Chem. Phys. 72, 2384 (1980).

6. P. Deymier and G. Kalonji, submitted to Acta Met.

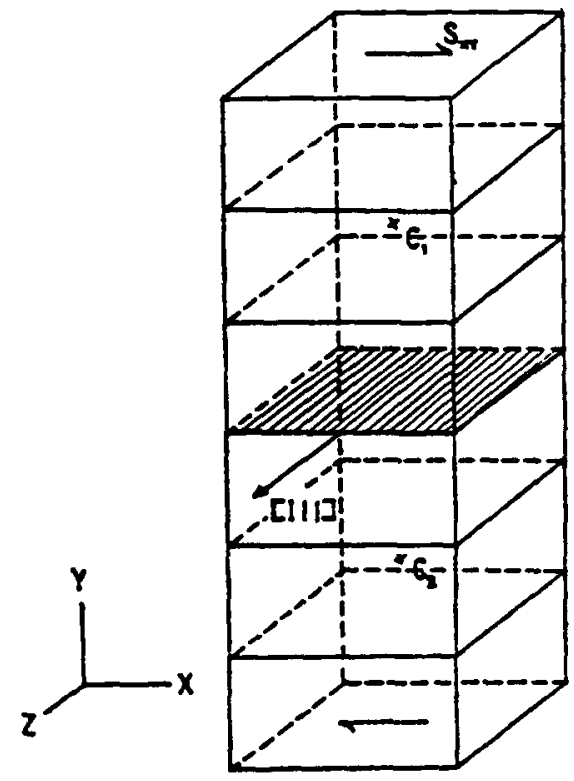

FIG. 1.

Schematic representation of the bicrystal under shear stress. The direction of the applied shear stress $S_{x y}$ is indicated. $C_{1}$ and $C_{2}$ are the centers of gravity of the slabs in the upper and lower grains. 


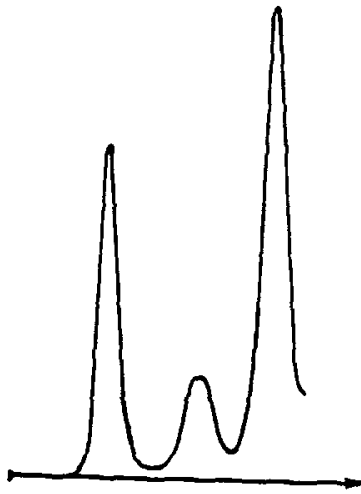

(a)

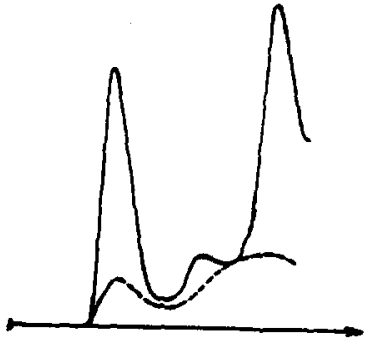

(b)

FIG. 2.

(a) The radial distribution functions of the crystalline bicrystal at $\mathrm{T}^{*}=0.06$. (b) The radial distribution of the bicrystal with a melted boundary at $\mathrm{T}^{*}=0.10$. We have superposed to the second radial distribution the radial distribution of a bulk liquid reduced to a scale of $1 / 6$.

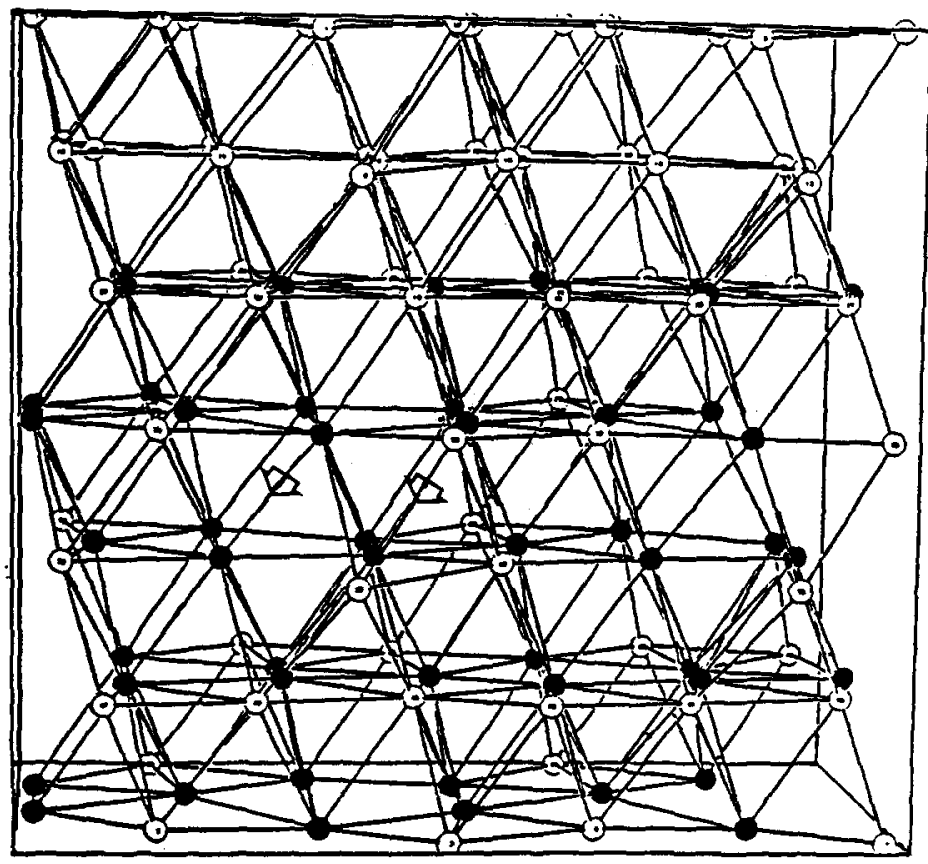

FIG. 3

Section of the crystalline bicrystal taken parallel to the grain boundary plane. The black atoms are some of the atoms located in the grain boundary. The arrows indicate the path of easy sliding. 


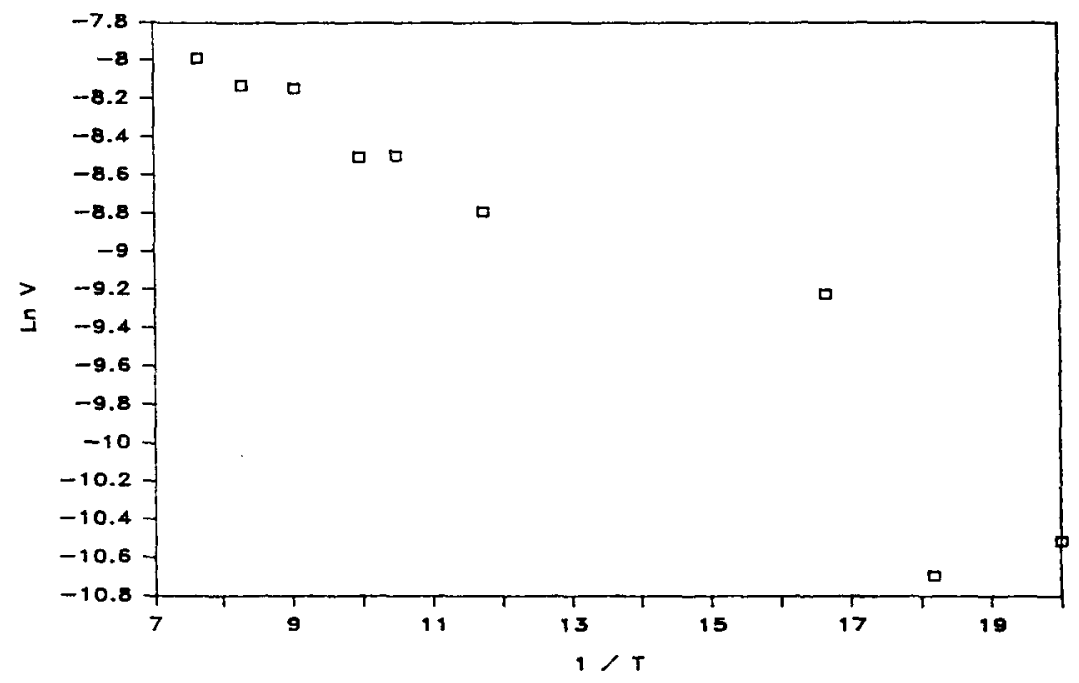

FIG. 4.

The temperature dependence of the logarithm of the sliding velocity. 